\title{
Identification and integrated analysis of CircRNA and miRNA of Radiation-Induced lung injury in a mice model
}

\section{Xi Yang}

Fudan University Shanghai Cancer Center

\section{Yida Li}

Fudan University Shanghai Cancer Center

\section{Liqing Zou}

Fudan University Shanghai Cancer Center

\section{Li Chu}

Fudan University Shanghai Cancer Center

\section{Luxi Ye}

Fudan University Shanghai Cancer Center

Jianjiao Ni

Fudan University Shanghai Cancer Center

\section{Xiao Chu}

Fudan University Shanghai Cancer Center

Tiantian Guo

Fudan University Shanghai Cancer Center

Zhengfei Zhu ( $\nabla$ fuscczzf@163.com )

Fudan University Shanghai Cancer Center https://orcid.org/0000-0001-7537-3619

\section{Research}

Keywords: circRNA, miRNA, radiation-induced lung injury, T cell proliferation

Posted Date: August 12th, 2020

DOI: https://doi.org/10.21203/rs.3.rs-56653/v1

License: (c) (i) This work is licensed under a Creative Commons Attribution 4.0 International License. Read Full License 


\section{Abstract}

Radiation-induced lung injury (RILI) is a main threat to patients received thoracic radiotherapy, it is of great importance to understand the molecular mechanism of RILI. Circular RNAs (CircRNAs) have been found to act as the regulator of multiple biological processes and circRNA-miRNA-mRNA axis could play an important role in signaling pathway of many human diseases including radiation injury, here, we first investigate the circRNA and miRNA of radiation-induced lung injury in a mice model. The mice received $12 \mathrm{~Gy}$ thoracic irradiation and the irradiated lung tissues at 48 hours after irradiation were analyzed by RNA Sequencing (RNA-seq) technique compared with normal lung tissues. We identified 21 significantly up-regulated while significantly 33 down-regulated miRNAs. Among 27 differentially expressed circRNAs, 10 were down-regulated and 17 were up-regulated. We then performed circRNAs GO analysis of the target mRNAs of these significantly differently expressed circRNAs. These differentially expressed miRNAs took part in series of cellular processes such as positive regulation of alpha-beta $T$ cell proliferation, interstitial matrix, collagen fibril organization, chemokine receptor activity, cellular defense response, and B cell receptor signaling pathway. Through this study, we found that immune-related molecular pathways play an important role in the early response after radiotherapy. In the future, research on the target mechanism and early intervention of circRNAs with associated miRNAs will benefit the treatment of RILI.

\section{Introduction}

Radiation-induced lung injury (RILI) is characterized by acute pneumonitis and chronic fibrosis, both of which can be lethal, occurring in approximately $10-20 \%$ of patients who undergo thoracic radiotherapy (1). Modern radiation therapy has witnessed evolution in radiation planning and delivery, successfully reducing the incidence of lung injury, however, it's still a troubling condition in clinical settings (2)\{!!! INVALID CITATION !!! , \#0;Briere, 2016 \#2\}. RILI is involved in a complex process, including varieties of molecular and cellular interactions which ultimately leading to large fibroblasts proliferation, accumulation, and differentiation, thus causing excessive deposition of extracellular matrix and pulmonary fibrosis (3).

The structure of Circular RNAs (CircRNAs) are covalently closed loops, which is different from linear RNAs. As a result, circRNAs do not contain either polyadenylated tail or 5'-3' polarity. CircRNAs could act as the regulator of multiple biological processes including cancer development, metabolism and immunity regulation (4). MicroRNAs (miRNAs) are small, highly conserved non-coding RNA molecules involved in the regulation of gene expression. Recent studies have reported that circRNAs can act as miRNAs sponges to compete for the same binding site of miRNAs, thus inhibiting miRNAs combining to their target mRNAs and suppressing the target genes (5). Various studies suggest that the circRNAmiRNA-mRNA axis is probably involved in signaling pathway of many human diseases by regulating pathogenicity-related gene expression, and the analysis of circRNA-related ceRNA networks has been performed in cancer (5), cardiology analysis (6) and even in radiation injury mice model (7). Luo et al. have been successfully investigate the circRNA-miRNA-mRNA pathways associated in radiation induced 
esophageal injury(RIEI) model, which successfully indicated sphingolipid metabolism might involved in RIEI process(7).

However, there are currently no reports focusing on circRNA-miRNA-mRNA network and pathways of intercellular communication in the RILI process. It is of great importance to understand the molecular mechanism by revealing circRNA-miRNA-mRNA competitive regulatory networks. As the recent improvement of RNA-sequencing (RNA-seq) technique facilitate obtaining huge and accurate results(8), RNA-seq become a proper way for us to detect the irradiation-responsive circRNAs in RILI. Therefore, in order to spot the potential role of circRNAs in RILI, we firstly performed an RNA-seq technique to detect irradiation-responsive genes in RILI mice model and compared the genome-wide expression between irradiated and normal lung tissue. The function of differentially expressed genes and circRNAs were also analyzed through gene ontology (GO) to identify pathways during intercellular communication in the RILI process. Taken together, these results could provide important resources and information for further RILI research.

\section{Materials And Methods}

\section{Animals}

The animal model of RILI was established according to previous studies $₫$ which have been verified by H\&E staining and Masson-staining $(9,10)$. Female Balb/c mice, 8 weeks, were purchased from Shanghai SLAC Laboratory Animal Co. Ltd. (Shanghai, China), Shanghai, China. Mice were housed five per cage and kept under standard laboratory conditions $\left(22 \pm 2^{\circ} \mathrm{C}, 55 \pm 10 \%\right.$ humidity, $12-12$ hours/light-dark cycle). All animal experimental procedures and protocols were conducted according to the guidelines of our institutional animal care and use committee and were approved with the reference number of 20150404A114 by Department of Laboratory Animal Science.

\section{Irradiation protocol}

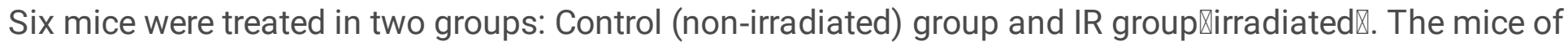
the control group only receive sham irradiation, while the mice of the IR group received thoracic irradiation. Radiation of mice was provided by Small Animal Radiation Research Platform (SARRP, FUSCC, Shanghai, China). All the six Mice were induced and maintained anesthesia with isopentane (increasing from $0.5 \%$ to $2.0 \%$ in $8 \mathrm{~min}$ for inducing and $1.5 \%$ for maintaining) and then were positioned with a self-designed box to fix their neck and stretch the body. For the IR group, the chest of each mouse was irradiated with a single dose of $12 \mathrm{~Gy}$ exposure using a $6 \mathrm{MV}$ linear accelerator (Siemens Primus-Hi) while for the control group each received $0 \mathrm{~Gy}$ exposure. The dose rate was $2 \mathrm{~Gy} / \mathrm{min}$. The source-surface distance was $1 \mathrm{~m}$, and the size of the radiation field was $2.5 \times 15 \mathrm{~cm}$. The head and the abdomen were shielded by $2.5 \mathrm{~cm}$ thick lead blocks to protect other body parts from irradiation. All mice were killed at day 3 after irradiation. Once a mouse was killed, the lung was taken and frozen in $-80^{\circ} \mathrm{C}$ fridge immediately. 


\section{H\&E staining and evaluation}

A part of lung samples from 3 irradiated and 3 control mice were processed to make paraffin blocks, then the tissue sections were deparaffinized by xylene and rehydration in a series of alcohol. After wash, tissue sections were processed as followed: $\quad 10 \mathrm{~min}$ haematine, 1-3s wash, 1-3s $1 \%$ Ethanol hydrochloride, 1030s wash, 1-3min 0.5\% eosin, 1-2s wash, 1-2s $80 \%$ alcohol, 2-3s $95 \%$ alcohol, 3-5s $95 \%$ alcohol, 5-10min $100 \%$ alcohol, $5-10 \mathrm{~min} x y l e n e$ carbonate, 2 min xylene (I), $2 \mathrm{~min}$ xylene (II), $2 \mathrm{~min}$ xylene (III). After drying for some time, neutral balsam was used for mounting. The evaluation of the H\&E results were performed according to the previous reported scoring system(11). The pneumonia alterations were defined as thickened alveolar walls, widening of the interlobular septa, alveolar septal edema and massive inflammatory cells infiltration in the alveoli. Three randomly selected fields of each mouse were scored by a pathologists in our hospital and scored as follow: 0 , no pneumonia; $1+$, pneumonia alterations in $1-20 \%$ of the field; $2+$, pneumonia alterations in $21-50 \%$ of the field; $3+$, pneumonia alterations in $51-100 \%$ of the field.

\section{RNA library construction and sequencing}

Lung samples were proceeded by Trizol (Invitrogen, Carlsbad, CA, USA) to isolate and purify total RNA. NanoDrop ND-1000 (NanoDrop, Wilmington, DE, USA) was used to quantify RNA amount and purity. RNA integrity was assessed using Agilent 2100 with RIN number $>$ 7.0. To deplete ribosomal RNA, Ribo-Zero ${ }^{\text {TM }}$ rRNA Removal Kit (Illumina, San Diego, USA) was used. The remaining RNAs were fragmented into small pieces by divalent cations at high temperature. Then, cDNAs were created by reverse-transcription of these cleaved RNA fragments. The cDNAs was then used for synthesizing U-labeled second-stranded DNAs with RNase H, E. coli DNA polymerase I, and dUTP. Then, an A-base is added to the blunt ends of each strand, preparing them for ligating to the indexed adapters, which each contains a T-base overhang for ligating the adapter to the A-tailed fragmented DNA. Single-or dual-index adapters are ligated to the fragments, and AMPureXP beads were used to perform size selection. After the using heat-labile UDG enzyme on the U-labeled second-stranded DNAs, PCR was proceeded with following settings: 3 mins, 95 ${ }^{\circ} \mathrm{C}$ for initial denaturation; 15 secs, $98^{\circ} \mathrm{C}$ for 8 cycles of denaturation; 15 secs, $60^{\circ} \mathrm{C}$ for annealing; 30 secs, $72^{\circ} \mathrm{C}$ for extension; 5 mins, $72^{\circ} \mathrm{C}$ for final extension. The insert size of the final cDNA library was proximately 300 bp in average. Finally, Illumina Hiseq 4000 (LC Bio, China) was used to perform pairedend sequencing.

The quantity and purity of each RNA sample was verified using Bioanalyzer 2100 with RIN number $>7.0$ and RNA 6000 Nano LabChip Kit (Aglient, Santa Clara, CA, USA) with 28S/18S ratio between 1.8 and 2.2. Approximately $1 \mu \mathrm{g}$ of total RNA was used to prepare miRNA library according to the protocol of TruSeq Small RNA Sample Prep Kits (Illumina, San Diego, USA).

\section{Differential expression analysis of mRNA, circRNAs and miRNAs}

Cutadapt (12) was used to remove the reads with low quality or undetermined bases and adaptor contamination. After verifying sequence quality by FastQC 
(http://www.bioinformatics.babraham.ac.uk/projects/fastqc/), Bowtie2 (13) and topaht2 (14) were used to map reads to the genome of mice. Remaining reads (unmapped reads) were still mapped to genome using tophat-fusion. CIRCExplorer was used to denovo assemble the mapped reads to circRNAs at first; Then, back splicing reads were identified in unmapped reads by tophat-fusion and CIRCExplorer. All samples were generated unique circRNAs. CircRNA expressions from different samples or groups were caculated by scripts in house. The differentially expression was defined as an srpbm log2 (fold change) $>1$ or log 2 (fold change) $<-1$ along with $p$ value $₫ 0.05$ by $R$ package - EdgeR.

As for miRNAs, raw reads were subjected to ACGT101-miR (LC Sciences, Houston, Texas, USA) to remove low complexity, junk, adapter dimers, common RNA families (snRNA, snoRNA, rRNA, tRNA,) and repeats. Subsequently, known miRNAs and novel miRNAs were identified in miRbase 22.0 using BLAST search. Finally, we defined differential expressed miRNAs according to normalized deep-sequencing counts by Chi-squared $n X n$ test, Fisher exact test, Student $t$ test, Chi-squared $2 X 2$ test, or ANOVA with a $p$ value less than 0.05 .

\section{circRNA-miRNA-mRNA network construction and functional analysis}

To explore the function of circRNAs as ceRNAs, we predicted the target miRNAs of circRNAs using MiRanda (3.3a) and Targetscan (5.0) according to the protocol of softwares with a maximum binding free energy less than -20 along with a Targetscan score more than 50 . After the prediction, miRNAs which could bind with differentially expressed circRNAs were selected to further predict the target mRNAs. Then, the significantly differentially expressed gene symbols were mapped into the terms in GO database and pathways in KEGG database. Based on this, 4 parameters were calculated including S (significantly differentially expressed gene symbols of certain GO term or pathway), TS (total gene symbols that are significantly differentially expressed), B (total gene symbols of certain GO term or pathway), TB (total gene symbols), then hypergeometric test was performed to find the most significantly enriched terms or pathways compared with the genome background. Then, Cytoscape3.5.1 was used to display the circRNA-miRNA-mRNA networks.

\section{Statistics}

FPKM (15) were calculated by StringTie to quantify the expression level of mRNAs and circRNAs. The differentially expression was defined as an FPKM log2 (fold change) $>1$ or log2 (fold change) <-1 along with $p$ value 0.05 by $R$ package - Ballgown (16). Differentially expressed miRNAs were defined according to normalized deep-sequencing counts by Chi-squared $n X n$ test, Fisher exact test, Student $t$ test, Chisquared $2 X 2$ test, or ANOVA with a p value less than 0.05 .

\section{Results}

Histologic evaluation of acute radiation-induced lung injury 
Using H\&E staining, lung tissue from radiated mice at $48 \mathrm{~h}$ after radiation was compared with control group to identify the model of radiation-induced lung injury. As described in method, 3 randomly selected fields of each mouse were evaluated. For the 9 fields of 3 mice in IR group, 7 were scored $3+$ while the other 2 fields had a score of $2+$. As for control group, all the 9 fields were scored 0 . As the representative images shown in Figure 1, compared to control group, irradiated lungs showed thickened alveolar walls, widening of the interlobular septa, alveolar septal edema and massive inflammatory cells infiltration in the alveoli. These pathological changes is consistent of previous report of acute RILI induced by a single dose of 12 Gy chest radiation (17).

\section{Expression profile of circRNAs}

According to the methods previously described, we detected 7103 circRNAs in the six mice in total. In order to find out the changes of circRNAs after RILI, we analyzed the differently expressed circRNAs with a cutoff fold changes $\geq 2$ or $\leq 0.5$ along with $p<0.05$. As a result, 27 significantly dysregulated circRNAs were found. Among these circRNAs, 17 were up-regulated significantly while 10 were down-regulated significantly. The results were shown as Volcano plots and heatmaps to visually show the expression profile differences of RILI from normal lung (Figure 2).

\section{Differently expressed miRNAs}

After acquiring the sequencing data of miRNAs, 785 miRNAs were detected in total in two groups. 73 miRNAs were only detected in control group while 60 miRNAs were only detected in RILI group (Supplementary Figure 1). Then, we analyzed the differently expressed miRNAs as described previously with a $\mathrm{p}$ value $\mathbb{0} .05$. Among these dysregulated miRNAs, 21 were up-regulated significantly while 33 were down-regulated significantly. Volcano plots and heatmaps were also displayed to visually understand the expression profile differences of RILI from normal lung (Figure 3). Besides, the top five up- and dowuregulated circRNAs and miRNAs were listed in the Table 1 and Table 2.

\section{GO and KEGG pathway analysis in these circRNAs functioned as ceRNAs}

To further investigate the potential functions of the dysregulated circRNAs via ceRNA networks $₫$ we next performed series of anylasis to predict the respective sponge miRNAs and target mRNA of all the dysregulated circRNAs. A GO analysis of the target mRNAs of these significantly differently expressed circRNAs was performed. As shown in Figure 4A, positive regulation of alpha-beta T cell proliferation, interstitial matrix, collagen fibril organization, chemokine receptor activity, cellular defense response, and $B$ cell receptor signaling pathway were the main functions associated with dysregulated circRNAs.

We next performed KEGG pathway analysis for these target mRNAs of dysregulated circRNAs (Figure 4B). The main enriched pathways include: Th17 cell differentiation, Th1 and Th2 cell differentiation, primary immunodeficiency, leishmaniasis, and hematopoietic cell lineage.

CeRNA networks were constructed based on screened mRNAs and bioinformatics prediction 
To better understand the relationship between these circRNAs and RILI, differentially expressed mRNAs were filtered from mRNAs of Th1 and Th2 differentiation pathways in KEGG analysis to construct a circRNA-miRNA-mRNA network. After filtering, a total of 105 cirRNA-miRNA-mRNA pathways were constructed including 16 cirRNAs, 26 miRNAs, and 22 mRNAs. Considering the complexity in this ceRNA network, we constructed a visually cirRNA-miRNA-mRNA network according to the filtered RNAs (Figure 5). It may help us better understand the interaction among RNAs and further investigate the mechanisms in the process of RILI.

\section{Discussion}

In this work, we firstly investigated irradiation-induced changes at the level of transcription of miRNAs and circRNAs in irradiated lung tissues and non-irradiated lung tissues by RNA-seq. By comparison of the transcriptome profiles in response to irradiation, we identified 21 significantly up-regulated while significantly 33 down-regulated miRNAs. Among 27 differentially expressed circRNAs, 10 were downregulated and 17 were up-regulated. We then performed circRNAs GO analysis of the target mRNAs of these significantly differently expressed circRNAs. These differentially expressed miRNAs took part in series of cellular processes, for example, positive regulation of alpha-beta T cell proliferation, interstitial matrix, collagen fibril organization, chemokine receptor activity, cellular defense response, and B cell receptor signaling pathway.

Previous studies have revealed that after inducing lung injury, T cell proliferation could be inhibited. In vitro studies have found that in alveolar liquid extracted from patients with acute hypersensitivity pneumonitis, lymphocyte proliferation was limited, including T cells (18). Additionally, some studies have shown part of the underlying mechanisms, an in vivo study has demonstrated that after physical injury, intracellular arginine in T cells is decreased, therefore causing inhibition of proliferation (19). As for collagen fibril organization, many studies have focused on the relationship between lung injury and collagen fibril formation or remodeling. For example, Tager et al. have provided evidence that lung fibrosis is mediated by lysophosphatidic acid (LPA) after injury (20). On the other hand, injury of alveolar epithelial type II cells (AE2 cells) is tightly correlated with fibrotic remodeling (21). Interstitial matrix is associated with lung injury as well. Including collagen organization mentioned above, some mechanisms after lung injury have also been reported to be associated with changes of interstitial matrix. In acute lung injury, restoration and thickening of the interstitial matrix has been reviewed (22). And the pathological characteristics of the interstitial compartment following irradiation were also listed and reviewed (23). Based on our GO results of the differentially expressed miRNAs, these three functions stood out and interestingly, are related to lung injury, which are consistent with previous studies above. With the help of our RNA profile results, the underlying mechanisms could be further investigated.

CircRNAs were reported to have functions such as sequestration of microRNAs or proteins, modulation of transcription and interference with splicing, and even translation to produce polypeptides(24). The functions and clinical impacts of ceRNAs were reported in varies diseases which may present opportunities for new therapeutic approaches(25). In recent years, microRNA and circRNA sequencing 
techniques were broadly used to recognize potential targets from the complex RNA networks based on the models of varies disease(26-28). Sequencing of circRNAs were even performed in the RIEI mice model and sepsis-induced acute lung injury(29), these previous studies have successfully provided meaningful potential target or mechanism for the diseases, respectively. To our knowledge, this is the first study analyzing the relationship of RILI and circRNAs by using RNA-seq data and computational approaches to identify the differentially expressed miRNAs and circRNAs. Previous studies have used similar methods to investigate the mechanism of radiation-induced esophageal injury. Additionally, our results provided potential clues to the mechanism of RILI. Since the roles of circRNAs in RILI have not yet been fully studied, this analysis could provide valuable data and information for future studies. Unlike many previous RILI studies on pulmonary fibrosis, this study found that $\mathrm{T}$ cell proliferation and differentiation pathway may be particularly important in the study of radiation pneumonia. Its association with circRNAs may be the focus of future research on circRNAs and radiation pneumonia. At the same time, with the increasingly development of immunotherapy in thoracic tumors (30), Immune-associated pneumonia and radiation pneumonia may play an important role in the study of RILI. The possible interaction of RILI also makes the study of immunity more important in RILI (2). Our results exactly demonstrated the dysregulation of ceRNAs after a series of crosstalk in the process of RILI, and these ceRNAs had potential in modulating immunology. However, more in-depth studies are needed due to the limited sample size in current research. Besides, since various lineage cells exist in lung tissues, further exploration of the relationship and molecular mechanism between cells, such as fibrocytes and immune cells, are needed in future study.

In conclusion, we have identified unique irradiation-induced miRNAs and circRNAs profile in RILI. The data also shows they have potential immunological functions during the pathogenesis of RILI. Moreover, our data could provide valuable resources and information for further studies, especially the roles of circRNAs in RILI. Based on the results of this study, the molecular function of associated circRNAs and further in-depth research such as immunomics analysis and Single cell sequencing should provide a more valuable resource and information for RILI research.

\section{References}

1. Huang, Y, Zhang, W, Yu, F, et al. The Cellular and Molecular Mechanism of Radiation-Induced Lung Injury. Med Sci Monit. 2017; 23: 3446-50.

2. Sakaguchi, M, Maebayashi, T, Aizawa, T, et al. Organizing pneumonia after thoracic radiotherapy followed by anti-PD-1 antibody treatment for patients with lung cancer: Three case reports. Thorac Cancer. 2019; 10(6): 1503-7.

3. Ding, NH, Li, JJ, Sun, LQ. Molecular mechanisms and treatment of radiation-induced lung fibrosis. Curr Drug Targets. 2013; 14(11): 1347-56.

4. Meng, S, Zhou, H, Feng, Z, et al. CircRNA: functions and properties of a novel potential biomarker for cancer. Mol Cancer. 2017; 16(1): 94. 
5. Rong, D, Sun, $\mathrm{H}, \mathrm{Li}, \mathrm{Z}$, et al. An emerging function of circRNA-miRNAs-mRNA axis in human diseases. Oncotarget. 2017; 8(42): 73271-81.

6. Zhang, $F$, Zhang, $R$, Zhang, $X$, et al. Comprehensive analysis of circRNA expression pattern and circRNA-miRNA-mRNA network in the pathogenesis of atherosclerosis in rabbits. Aging (Albany NY). 2018; 10(9): 2266-83.

7. Luo, J, Zhang, C, Zhan, Q, et al. Profiling circRNA and miRNA of radiation-induced esophageal injury in a rat model. Sci Rep. 2018; 8(1): 14605.

8. Zhao, Y, Zhang, J, Han, X, et al. Total body irradiation induced mouse small intestine senescence as a late effect. J Radiat Res. 2019; 60(4): 442-50.

9. Chen, J, Tian, X, Mei, Z, et al. The effect of the TLR9 ligand CpG-oligodeoxynucleotide on the protective immune response to radiation-induced lung fibrosis in mice. Mol Immunol. 2016; 80: 3340.

10. Traver, G, Mont, S, Gius, D, et al. Loss of Nrf2 promotes alveolar type 2 cell loss in irradiated, fibrotic lung. Free Radic Biol Med. 2017; 112: 578-86.

11. Szapiel, SV, Elson, NA, Fulmer, JD, et al. Bleomycin-induced interstitial pulmonary disease in the nude, athymic mouse. Am Rev Respir Dis. 1979; 120(4): 893-9.

12. M, M. Cutadapt removes adapter sequences from high-throughput sequencing reads. Embnet Journal. 2011; 17.

13. Langmead, B, Salzberg, SL. Fast gapped-read alignment with Bowtie 2. Nat Methods. 2012; 9(4): 357-9.

14. Kim, D, Pertea, G, Trapnell, C, et al. TopHat2: accurate alignment of transcriptomes in the presence of insertions, deletions and gene fusions. Genome Biol. 2013; 14(4): R36.

15. Trapnell, C, Williams, BA, Pertea, G, et al. Transcript assembly and quantification by RNA-Seq reveals unannotated transcripts and isoform switching during cell differentiation. Nat Biotechnol. 2010; 28(5): 511-5.

16. Frazee, AC, Pertea, G, Jaffe, AE, et al. Ballgown bridges the gap between transcriptome assembly and expression analysis. Nat Biotechnol. 2015; 33(3): 243-6.

17. Chen, J, Zhang, W, Zhang, L, et al. Glycyrrhetinic acid alleviates radiation-induced lung injury in mice. J Radiat Res. 2017; 58(1): 41-7.

18. Lesur, O, Mancini, NM, Janot, C, et al. Loss of lymphocyte modulatory control by surfactant lipid extracts from acute hypersensitivity pneumonitis: comparison with sarcoidosis and idiopathic pulmonary fibrosis. Eur Respir J. 1994; 7(11): 1944-9.

19. Zhu, ZF, Chen, HQ, Yu, WW, et al. Number of Negative Lymph Nodes is Associated with Survival in Thoracic Esophageal Squamous Cell Carcinoma Patients Undergoing Three-Field Lymphadenectomy. Annals Of Surgical Oncology. 2014; 21(9): 2857-63.

20. Ley, K, Zarbock, A. From lung injury to fibrosis. Nat Med. 2008; 14(1): 20-1. 
21. Birkelbach, B, Lutz, D, Ruppert, C, et al. Linking progression of fibrotic lung remodeling and ultrastructural alterations of alveolar epithelial type II cells in the amiodarone mouse model. Am J Physiol Lung Cell Mol Physiol. 2015; 309(1): L63-75.

22. Gill, SE, Yamashita, CM, Veldhuizen, RA. Lung remodeling associated with recovery from acute lung injury. Cell Tissue Res. 2017; 367(3): 495-509.

23. Multhoff, G, Vaupel, P. Radiation-induced changes in microcirculation and interstitial fluid pressure affecting the delivery of macromolecules and nanotherapeutics to tumors. Front Oncol. 2012; 2: 165.

24. Li, X, Yang, L, Chen, LL. The Biogenesis, Functions, and Challenges of Circular RNAs. Mol Cell. 2018; 71(3): 428-42.

25. Qi, X, Zhang, DH, Wu, N, et al. ceRNA in cancer: possible functions and clinical implications. J Med Genet. 2015; 52(10): 710-8.

26. Dasdag, S, Akdag, MZ, Erdal, ME, et al. Long term and excessive use of $900 \mathrm{MHz}$ radiofrequency radiation alter microRNA expression in brain. Int $J$ Radiat Biol. 2015; 91(4): 306-11.

27. Dasdag, S, Akdag, MZ, Erdal, ME, et al. Effects of $2.4 \mathrm{GHz}$ radiofrequency radiation emitted from WiFi equipment on microRNA expression in brain tissue. Int J Radiat Biol. 2015; 91(7): 555-61.

28. Pei, W, Tao, L, Zhang, LW, et al. Circular RNA profiles in mouse lung tissue induced by radon. Environ Health Prev Med. 2017; 22(1): 36.

29. Bao, X, Zhang, Q, Liu, N, et al. Characteristics of circular RNA expression of pulmonary macrophages in mice with sepsis-induced acute lung injury. J Cell Mol Med. 2019; 23(10): 7111-5.

30. Long, L, Zhao, C, Ozarina, M, et al. Targeting Immune Checkpoints in Lung Cancer: Current Landscape and Future Prospects. Clin Drug Investig. 2019; 39(4): 341-53.

\section{Tables}

Table 1. Top 5 significantly up- and down-regulated circRNAs 


\begin{tabular}{|llll|}
\hline CircRNA ID & Log2 fold change* & P value & regulation \\
\hline circRNA3975 & inf & $\varangle 0.01$ & up \\
\hline circRNA4510 & inf & 0.01 & up \\
\hline circRNA4584 & inf & 0.01 & up \\
\hline circRNA5229 & inf & 0.01 & up \\
\hline circRNA2066 & 3.31 & 0.02 & up \\
\hline circRNA640 & -inf & $\varangle 0.01$ & down \\
\hline circRNA1845 & -inf & 0.01 & down \\
\hline circRNA1204 & -3.86 & 0.01 & down \\
\hline circRNA744 & -3.07 & 0.01 & down \\
\hline circRNA15 & -inf & 0.02 & down \\
\hline
\end{tabular}

*inf means circRNAs only detected in radiation-induced lung injury group; -inf means circRNAs only detected in control group.

Table 2. Top 5 significantly up- and down-regulated miRNAs

\begin{tabular}{|c|c|c|c|}
\hline miRNA name & log2 fold change & $\mathrm{p}$ value & regulation \\
\hline mmu-miR-31-3p & -1.05 & $\varangle 0.01$ & down \\
\hline mmu-miR-181b-5p_R-1 & -0.54 & $₫ 0.01$ & down \\
\hline mmu-miR-146a-5p & -1.18 & $\varangle 0.01$ & down \\
\hline mmu-miR-872-3p & -0.50 & $\nabla 0.01$ & down \\
\hline mmu-miR-181c-3p & -0.85 & $\nabla 0.01$ & down \\
\hline mmu-miR-511-5p_L+1 & 1.15 & $\nabla 0.01$ & up \\
\hline mmu-miR-194-2-3p_R+1 & 1.88 & $\nabla 0.01$ & up \\
\hline mmu-miR-433-3p & 0.40 & $\nabla 0.01$ & up \\
\hline mmu-miR-25-3p & 0.41 & $\nabla 0.01$ & up \\
\hline mmu-miR-30a-3p & 0.55 & $\nabla 0.01$ & up \\
\hline
\end{tabular}


A

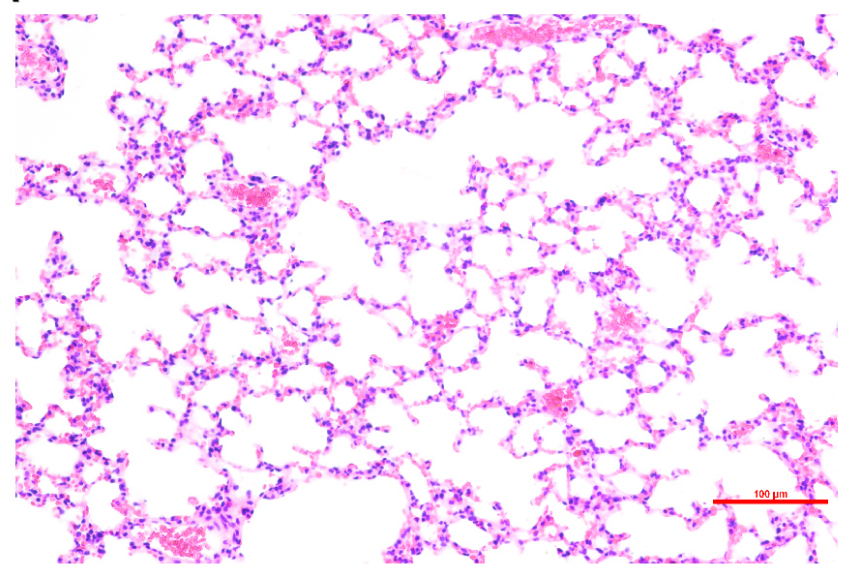

B

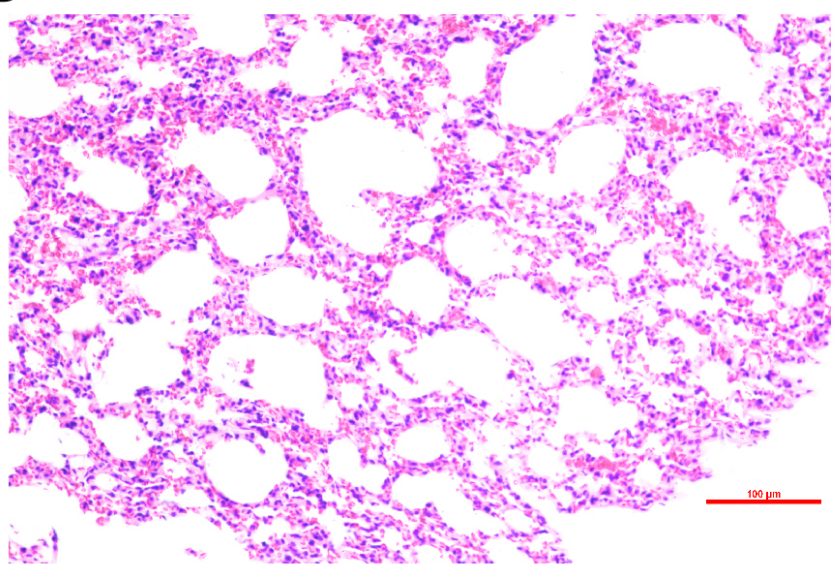

Figure 1

H\&E staining of lung tissue from: A) control group; B) IR group at 48h after radiation.

A

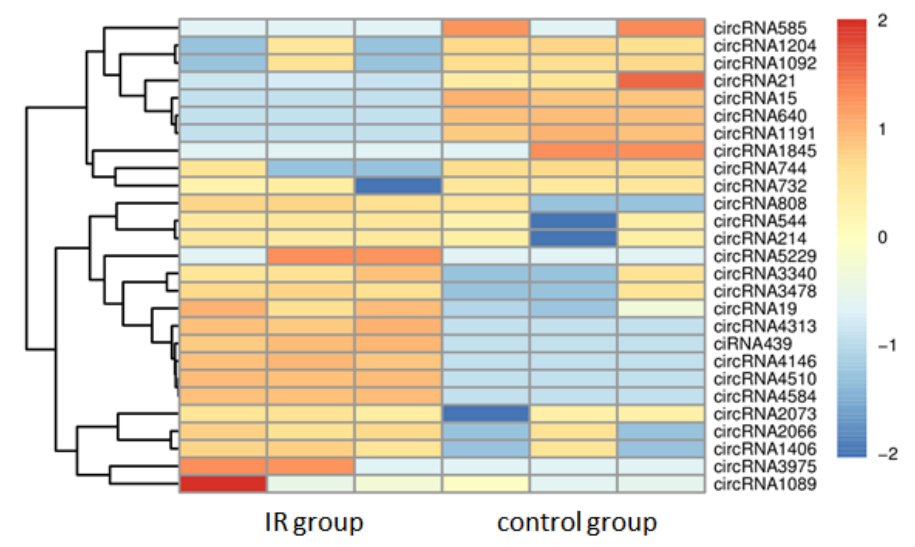

B

- up * no * down

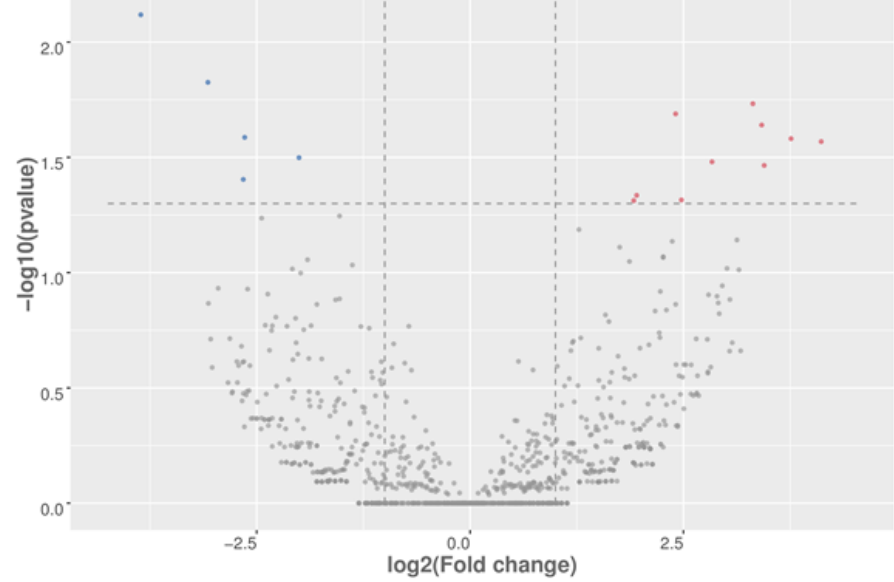

Figure 2

The circRNA profiling in radiation-induced lung injury group and control group. The A) heatmap and B) volcano plot displayed 17 up-regulated circRNAs and 10 down-regulated circRNAs. Red represents upregulation and blue represents downregulation. 
A

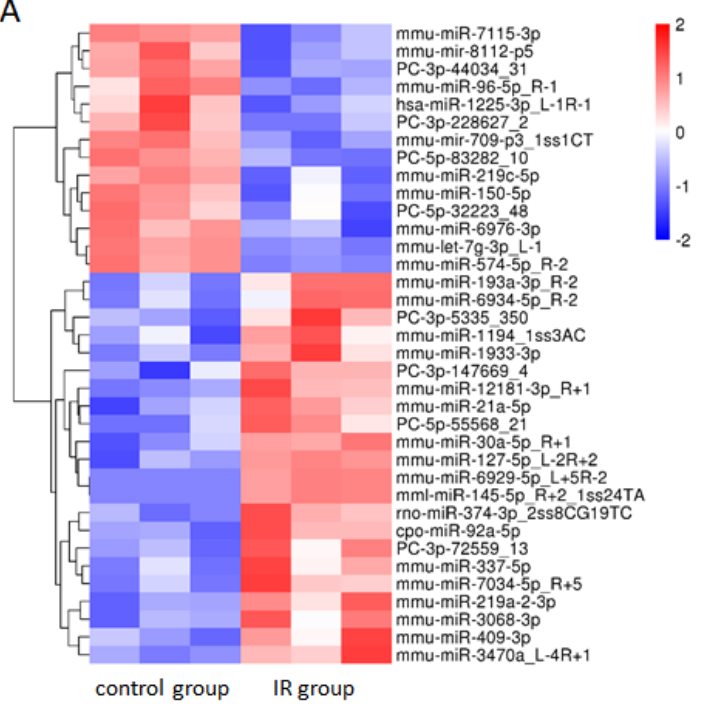

B

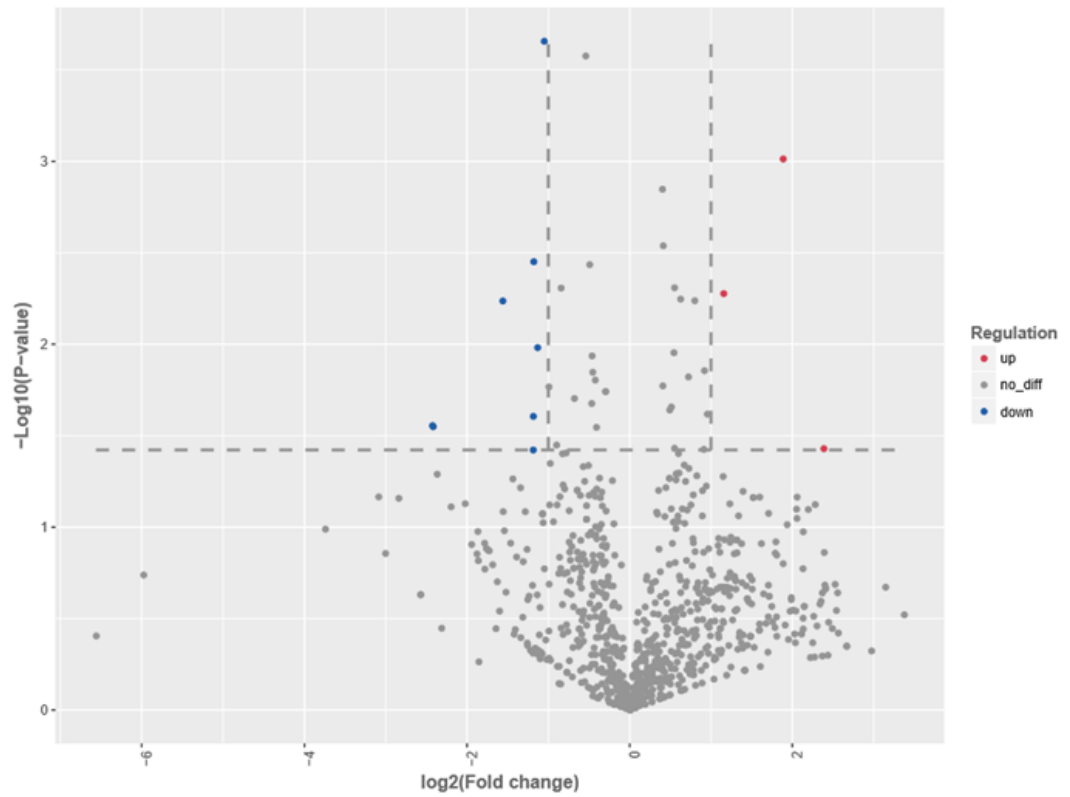

\section{Figure 3}

The miRNA profiling in radiation-induced lung injury group and control group. The A) heatmap and B) volcano plot displayed 21 up-regulated miRNAs and 33 down-regulated miRNAs. Red represents upregulation and blue represents downregulation.
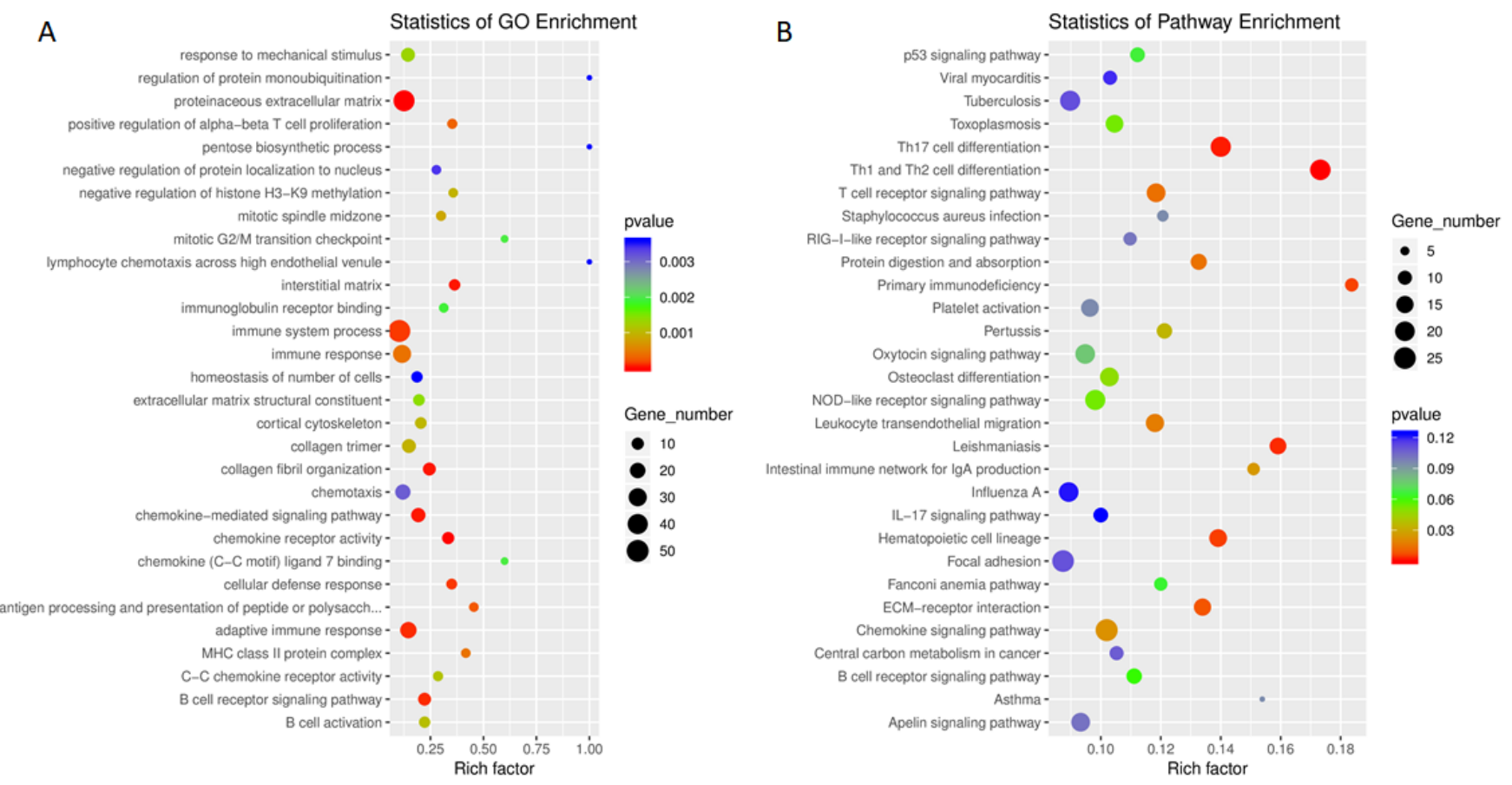

Figure 4 
A) GO annotations and B) KEGG pathway analysis for the mRNAs regulated by circRNA-miRNA-mRNA network. The top 20 according to $p$-value of each analysis are displayed.

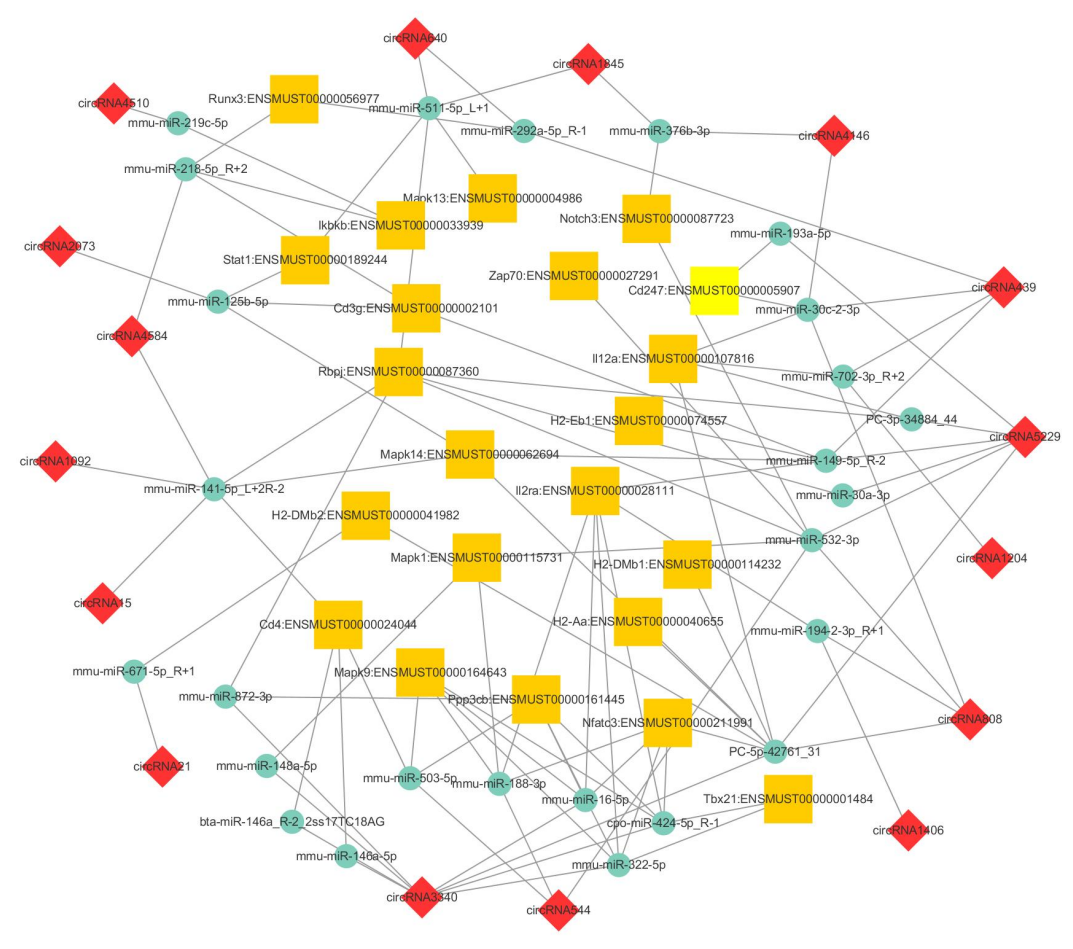

Figure 5

circRNA-miRNA-mRNA network based on the filtered mRNAs associated with collagen fibril organization function in $\mathrm{GO}$ analysis. The yellow square represents mRNAs, the green circle represents miRNAs, and the red diamond represents circRNAs. Their regulatory relationships are displayed as lines between them.

\section{Supplementary Files}

This is a list of supplementary files associated with this preprint. Click to download.

- SupplementaryFigure1.docx 